UDC 339.138:338.46

http://doi.org/10.21272/mmi.2019.3-05

JEL Classification: C38, I23, M31

Sergiy Kvitka,

D.Sc., Associate Professor, Ignacy Łukasiewicz Rzeszów University of Technology, Poland

Galina Starushenko,

D.Sc., Associate Professor, Ignacy Łukasiewicz Rzeszów University of Technology, Poland

Viktor Koval,

D.Sc., Odessa Institute of Trade and Economics of Kyiv National University of Trade and Economics, Ukraine

Hanna Deforzh,

D.Sc., Associate Professor, Volodymyr Vynnychenko Central Ukrainian State Pedagogical University in Kropyvnytskyi, Ukraine

Olha Prokopenko,

D.Sc., Professor, Tallinn University of Technology, Estonia; Collegium Mazovia Innovative University in Siedlce, Poland

\title{
MARKETING OF UKRAINIAN HIGHER EDUCATIONAL INSTITUTIONS REPRESENTATION BASED ON MODELING OF WEBOMETRICS RANKING
}

Abstract. The objective of the article is constructing of the two-factor model on the basis of the statistical material of the Webometric Rating of Universities, which analytically describes the status of Ukrainian higher educational institutions in terms of Webometrics indicators, provides an opportunity for its quantitative and qualitative analysis and forecasting of development trends of market educational services and marketing research in this area. The statistical analysis of the data of the Webometric Rating of Universities was conducted using the professional statistical information processing program STATGRAPHICS Centurion XV.I. Analytical relations are obtained using the Pade approximation technique. Mathematical editor Maple has been used to visualize the results of research and illustrate a qualitative picture of the rating of Ukrainian universities in the Internet space using the Webometric Indicators system. A two-factor model of the state and forecast of academic representation of Ukrainian universities according to Webometric rating is constructed. Using the proposed model, an analytical expression is obtained that allows for quantitative and qualitative assessments of the world ranking of higher educational institutions of Ukraine in the Webindicators system Webometrix in order to increase their academic presence on the Internet, to strengthen the international authority and raise the national science school as a whole for a qualitatively new degree of development. The scientific novelty of the work is in the inclusion in the model of the time factor, the availability of which makes it possible, firstly, to extend the statistical material used in time and, secondly, to predict the trends and prospects of presentation in the Internet space of Ukrainian universities as advanced research centers. The main results of the work can be useful as a methodological material during the educational process in higher educational institutions, in the training and improvement of the skills of management personnel, in developing programs for reforming the educational process in higher education in order to bring it closer to European requirements and achieve the level of the best world universities. The presented methodology and data analysis algorithm can be generalized to evaluate rating systems of other nature: when evaluating students' educational and community work, financial analysis, in particular, to assess the probability of default of non-lending organizations, to determine the reliability of commercial banks, to conduct sociological tests, and surveys, medical and biological research and other areas of public administration.

Keywords: higher educational institution, university, Webometric rating, Pade approximation, two-factor model.

Introduction. The Law of Ukraine "On Higher Education" (2014) states that state policy in the field of higher education is based on the principles of international integration and integration of the higher education system of Ukraine into the European Higher Education Area, provided that the preservation and development of the achievements and progressive traditions of the national higher education. In this context, the vector of modern trends in higher education in Ukraine is oriented towards the entry of the country into the educational and scientific space of Europe and the world, the modernization of educational activities in the context of the European canons, the development of programs for the modernization of

Cite as: Kvitka, S., Starushenko, G., Koval, V., Deforzh, H., \& Prokopenko, O. (2019). Marketing of Ukrainian higher educational institutions representation based on modeling of Webometrics Ranking. Marketing and Management of Innovations, 3, 6072. http://doi.org/10.21272/mmi.2019.3-05 
the training of high-level specialists, competitive in the world labor market. The implementation of these areas will contribute to embedding global value chains (Koval et al., 2019). That is why the trend of recent years for advanced Ukrainian universities has been to analyze the results of their participation in significant international and national ratings.

Webometrics rating is one of the most well-known and authoritative academic ratings of higher education institutions. Since 2004, every six months, Spanish National Research Council (CSIC), Spanish National Research Council (Cybermetrics Lab) under the Ministry of Science and Innovation in Spain, conducts an independent, objective, free, and open-minded assessment of universities from all over the world to provide a reliable, multidimensional, updated and useful information about their work, based on the representation and influence in the Internet space (Ranking Web of Universities, 2019).

Webometric rating is estimated by more than 24 thousand universities of the world, including 323 Ukrainian, and published twice a year - in July and January. According to the authors, the Webometrix rating with the help of independent Web indicators and scientifically grounded model indirectly evaluates all aspects of the functioning of universities: scientific activity, educational work, development of culture, social status, sports achievements, and the very versatility distinguishes Webometrics from other Internet ratings. However, the most important in the rating are the figures of the citation of scientific publications of universities on the Internet and their representation in the database Scopus. The rating also positively takes into account the level of openness at the university, that is, the possibility of open access to the results of their scientific research, educational and methodological materials, normative base, etc.

Literature Review. The investigation of the quality factors of research and education activities of domestic higher educational institutions according to influential international ratings, as well as the analysis of evaluation indicators, are becoming more relevant in the context of European integration processes in Ukraine in modern times. This is due to the considerable interest of Ukrainian researchers in studying this problem. Thus, the work of Harvey (2008), Shin \& Toutkoushian (2011), Westerheijden (2012), Porzionato \& De Marco (2015), Kochetkova et al. (2016) are devoted to the analysis of modern ratings of Higher education as a tool for assessing the quality of higher education. In the article by Morze \& Buinytska (2015), the issue of creating an open electronic environment as one of the most important tasks of universities in terms of improving the quality of education was considered. In the articles by Buinytska et al. (2013), Marginson, S. (2014) provides a comprehensive analysis of indicators of the Webometric rating and their impact on the generalized place of higher education in the ranking.

A generalized multidimensional correlation model of the dependence of the general rating of universities of Ukraine on the web indicators of Webometrics, based on the statistical material of the Webometric Ratings of Universities in January 2018, is presented in the work of Starushenko \& Bazylevskyi (2018).

The publication of Novak et al. (2019) analyzed the correspondence of master's programs in higher educational institutions of the United States, the European Union and Ukraine, and based on the obtained results the recommendations were given regarding the modernization of master's programs in Ukraine up to standards of the European Higher Education Area. In the article by Nelipa \& Batrymenko (2018), a strategy has been developed based on a competent approach, a management model is constructed, and an educational and scientific program of training civil servants in graduate school is presented. Rudenko et al. (2018) investigated in the scientific paper the peculiarities of the management of international educational projects between the universities of Poland and Ukraine, proposed to organize the management of international projects in the field of higher education and science through creation of independent management and financial aspects of joint educational and research centers, with the involvement of non-state scientific and educational organizations.

In the framework of the subject under consideration, it is expedient, in our opinion, to study the issue that was not yet adequately covered in the literature, namely, the construction of a two-factor model, which, 
according to the Webometric rating, analytically describes at the time the representation of Ukrainian universities in the Internet space. Such a model will make it possible to calculate the forecast for the ranking of Ukrainian universities for Webometric indicators and will allow, if necessary, to correct the vector of scientific activity of domestic universities in order to improve the results of this or that Web indicator.

The objective of the article is the construction of the Webometric Rating of Universities of the twofactor model on the basis of the statistical material (Koval et al., 2018), which analytically describes the status of Ukrainian higher educational institutions in terms of Webometrics indicators, provides an opportunity for its quantitative and qualitative analysis and forecasting of development trends.

Methodology and research. The initial data for the construction of a two-factor model that describes the state, patterns and trends of the world ranking of Ukrainian universities in the Internet space by the Webometric Indicators system in time was a sample of 50 best-rated Ukrainian universities in the 20182019 period: in January 2018 (New Ranking Webometrics, 2018a), in July 2018 (New Ranking Webometrics, 2018b) and in January 2019 (Ranking Web of universities, 2019).

From the mathematical point of view, the construction of the desired model, illustrating the dependence of the ranking on two parameters - the place (number) of the university in the rating scale and the time parameter - leads to the need to construct the function of two variables:

$$
z=z(y, t)
$$

where $y=y(x), x$-a serial number of the university in the rating; $t$ - time parameter.

Ukrainian universities that are part of the top 50 Ukrainian universities according to the Webometrix rating are re-coded according to the order (rank, ordinal scale), namely: $x_{i}=i, i=1,2, \ldots, 50$; with the higher serial number corresponding to the higher (worst) place of the university in the ranking.

The values of the time factor are determined as follows: $t=0-$ January 2018; $t=6-$ July 2018 and $t=12$ - January 2019.

All statistical procedures used in constructing the model were performed in the program STATGRAPHICS Centurion XV.I.

Results. Using the STATGRAPHICS Simple Regression tool, we will construct the regressive dependence of the resultant attributes $y_{j}, j=1,2,3$ (the world ranking of Ukrainian universities in January 2018 - July 2018 - January 2019 ) from ordinary factors. The results of the calculations will be placed according to the table $1-3$.

Summarizing conclusions based on the estimated data in Table 1-3 we will reduce in Table 4.

To write the expression of the desired function of the form (1), we use the Pade approximation method (Baker \& Graves-Morris, 1996). If the function $f(t)$ can be represented as a power series:

$$
f(t)=\sum_{i=0}^{\infty} c_{i} t^{i}
$$

then the Pade approximation is called the rational function of the form:

$$
f_{[L / M]}(t)=\frac{a_{0}+a_{1} t+\ldots+a_{L} t^{L}}{b_{0}+b_{1} t+\ldots+b_{M} t^{M}}
$$

the decomposition of which in a Maclaurin series coincides with expansion (5), as long as it is possible.

The function of type (3) has $L+1$ coefficients in the numerator and $M+1-$ in denominator, and without limitation of the general nature of considerations can be put $b_{0}=1$. 
S. Kvitka, G. Starushenko, V. Koval, H.Deforzh, O., Prokopenko. Marketing of Ukrainian higher educational institutions representation based on modeling of Webometrics Ranking

Table 1. The regression model of the world ranking of Ukrainian universities in January 2018 Simple Regression - World Rank January 2018 vs. Ukraine Rank January 2018

Dependent variable: World Rank January 2018

Independent variable: Ukraine Rank January 2018

Square root- $X$ model: $Y=a+b^{*} s q r t(X)$

\section{Coefficients}

\begin{tabular}{|l|l|l|l|l|}
\hline & Least Squares & Standard & $T$ & \\
\hline Parameter & Estimate & Error & Statistic & P-Value \\
\hline Intercept & 570.567 & 47.2868 & 12.0661 & 0.0000 \\
\hline Slope & 822.423 & 9.36418 & 87.8265 & 0.0000 \\
\hline
\end{tabular}

\section{Analysis of Variance}

\begin{tabular}{|l|l|l|l|l|l|}
\hline Source & $\begin{array}{l}\text { Sum of } \\
\text { Squares }\end{array}$ & Df & $\begin{array}{l}\text { Mean } \\
\text { Square }\end{array}$ & F-Ratio & -Value \\
\hline Model & $8.9443 \mathrm{E} 7$ & 1 & $8.9443 \mathrm{E} 7$ & 7713.50 & 0.0000 \\
\hline Residual & 556591. & 48 & 11595.6 & & \\
\hline Total (Corr.) & $8.99996 \mathrm{E} 7$ & 49 & & & \\
\hline
\end{tabular}

Correlation Coefficient $=0.996903$

R-squared $=99.3816$ percent

R-squared (adjusted for d.f.) $=99.3687$ percent

Standard Error of Est. $=107.683$

Mean absolute error $=87.9665$

Durbin-Watson statistic $=0.753736(\mathrm{P}=0.0000)$

Lag 1 residual autocorrelation $=0.606288$

Plot of Fitted Model

World Rank January $2018=570,567+822,423^{*}$ sqrt(Ukraine Rank January 2018)

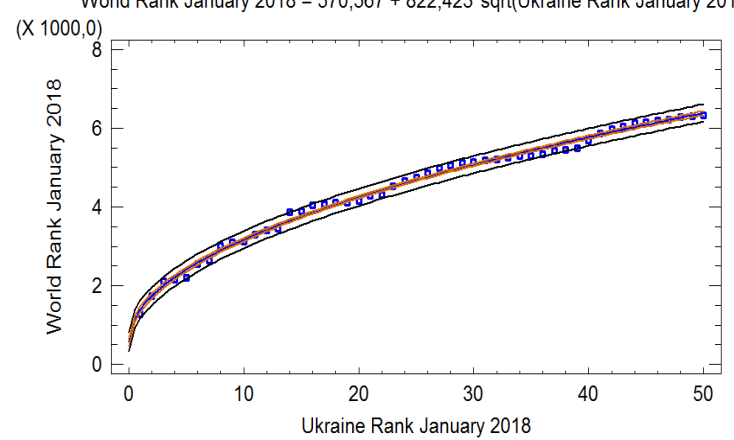

\begin{tabular}{|c|c|c|}
\hline Model & Correlation & R-Squared \\
\hline Square root- $X$ & 0.9969 & $99.38 \%$ \\
\hline Multiplicative & 0.9967 & $99.35 \%$ \\
\hline Squared-Y & 0.9958 & $99.16 \%$ \\
\hline \begin{tabular}{|l|} 
Double square \\
root
\end{tabular} & 0.9905 & $98.10 \%$ \\
\hline $\begin{array}{l}\text { Square root-Y } \\
\text { logarithmic-X }\end{array}$ & 0.9880 & $97.61 \%$ \\
\hline \begin{tabular}{|l|} 
Squared-Y \\
square root-X
\end{tabular} & 0.9854 & $97.10 \%$ \\
\hline Linear & 0.9767 & $95.39 \%$ \\
\hline $\begin{array}{l}\text { Logarithmic-Y } \\
\text { square root-X }\end{array}$ & 0.9720 & $94.48 \%$ \\
\hline Logarithmic-X & 0.9700 & $94.10 \%$ \\
\hline Double squared & 0.9582 & $91.81 \%$ \\
\hline Square root-Y & 0.9527 & $90.77 \%$ \\
\hline \begin{tabular}{|l|} 
Double \\
reciprocal
\end{tabular} & 0.9292 & $86.35 \%$ \\
\hline \begin{tabular}{|l|} 
Squared-Y \\
logarithmic-X
\end{tabular} & 0.9200 & $84.64 \%$ \\
\hline Exponential & 0.9162 & $83.94 \%$ \\
\hline Squared-X & 0.9025 & $81.45 \%$ \\
\hline $\begin{array}{l}\text { Square root-Y } \\
\text { squared-X }\end{array}$ & 0.8600 & $73.96 \%$ \\
\hline S-curve model & \begin{tabular}{|l|l|} 
\\
\end{tabular} & $67.05 \%$ \\
\hline $\begin{array}{l}\text { Logarithmic-Y } \\
\text { squared-X }\end{array}$ & 0.8062 & $65.00 \%$ \\
\hline Reciprocal-Y & -0.8026 & $64.42 \%$ \\
\hline Reciprocal-X & -0.6984 & $48.78 \%$ \\
\hline \begin{tabular}{|l} 
Reciprocal-Y \\
squared-X
\end{tabular} & -0.6676 & $44.57 \%$ \\
\hline \begin{tabular}{|l|} 
Squared-Y \\
reciprocal-X
\end{tabular} & -0.5965 & $35.58 \%$ \\
\hline $\begin{array}{l}\text { Reciprocal-Y } \\
\text { square root-X }\end{array}$ & $<$ no fit $>$ & \\
\hline \begin{tabular}{|l|} 
Reciprocal-Y \\
logarithmic-X
\end{tabular} & $\mid<n o$ fit $>$ & \\
\hline $\begin{array}{l}\text { Square root-Y } \\
\text { reciprocal-X }\end{array}$ & $\mid<$ no fit $>$ & \\
\hline \begin{tabular}{|l|} 
Logistic \\
\end{tabular} & $\mid<$ no fit $>$ & \\
\hline Log probit & $<1$ & \\
\hline
\end{tabular}

Sources: compiled by authors. 
S. Kvitka, G. Starushenko, V. Koval, H. Deforzh, O. Prokopenko. Marketing of Ukrainian higher educational institutions representation based on modeling of Webometrics Ranking

Table 2. The regression model of the world ranking of Ukrainian universities in July 2018

\begin{tabular}{|c|c|c|c|c|}
\hline \multicolumn{5}{|c|}{$\begin{array}{c}\text { Simple Regression - World Rank July } 2018 \\
\text { vs. Ukraine Rank July } 2018\end{array}$} \\
\hline \multicolumn{5}{|c|}{$\begin{array}{l}\text { Dependent variable: World Rank July } 2018 \\
\text { Independent variable: Ukraine Rank July } 2018 \\
\text { Square root-X model: } Y=a+b^{*} \text { sqrt }(X)\end{array}$} \\
\hline \multicolumn{5}{|c|}{ Coefficients } \\
\hline & Least Squares & Standard & $T$ & \\
\hline \begin{tabular}{|l|} 
Parameter \\
\end{tabular} & Estimate & Error & Statistic & $P$-Value \\
\hline Intercept & 516.728 & 52.3765 & 9.86565 & 0.0000 \\
\hline Slope & 792.407 & 10.3721 & 76.398 & 0.0000 \\
\hline
\end{tabular}

Analysis of Variance

\begin{tabular}{|l|l|l|l|l|l|}
\hline Source & $\begin{array}{l}\text { Sum of } \\
\text { Squares }\end{array}$ & $D f$ & $\begin{array}{l}\text { Mean } \\
\text { Square }\end{array}$ & F-Ratio & $P$-Value \\
\hline Model & $8.30332 \mathrm{E} 7$ & 1 & $8.30332 \mathrm{E} 7$ & 5836.65 & 0.0000 \\
\hline Residual & 682856. & 48 & 14226.2 & & \\
\hline $\begin{array}{l}\text { Total } \\
\text { (Corr.) }\end{array}$ & $8.37161 \mathrm{E} 7$ & 49 & & & \\
\hline
\end{tabular}

Correlation Coefficient $=0.995913$

R-squared $=99.1843$ percent

R-squared (adjusted for d.f.) $=99.1673$ percent

Standard Error of Est. $=119.274$

Mean absolute error $=87.7746$

Durbin-Watson statistic $=0.646155(P=0.0000)$

Lag 1 residual autocorrelation $=0.675805$

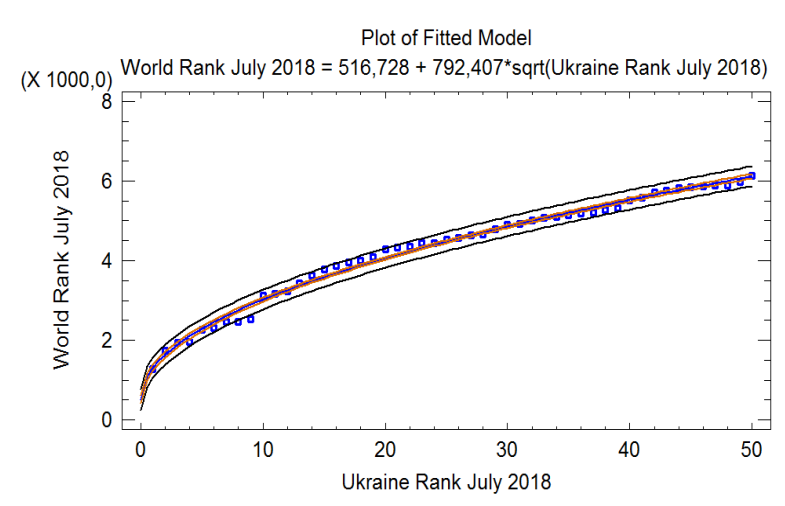

Comparison of Alternative Models

\begin{tabular}{|l|l|l|}
\hline Model & Correlation & $R$-Squared \\
\hline
\end{tabular}

\begin{tabular}{|l|l|l|}
\hline Square root-X & 0.9959 & $99.18 \%$ \\
\hline
\end{tabular}

\begin{tabular}{|l|l|l|}
\hline Squared-Y & 0.9955 & $99.10 \%$ \\
\hline Mulpative & 0.9936 & $98.73 \%$ \\
\hline
\end{tabular}

\begin{tabular}{|l|l|l|}
\hline Multiplicative & 0.9936 & $98.73 \%$ \\
\hline
\end{tabular}

\begin{tabular}{|l|l|l|}
\hline Double square & 0.9890 & $97.80 \%$
\end{tabular}

root

\begin{tabular}{l|l|l|}
\hline Squared-Y & 0.9885 & $97.72 \%$ \\
\hline
\end{tabular}

\begin{tabular}{l|l|l} 
square root- $X$ & & \\
\hline Square root-Y & 0.9852 & $97.07 \%$
\end{tabular}

\begin{tabular}{l|l|l|}
$\begin{array}{l}\text { Square root-Y } \\
\text { logarithmic-X }\end{array}$ & 0.9852 & $97.07 \%$ \\
\hline Linear & 0.9733 & $94.74 \%$ \\
\hline
\end{tabular}

\begin{tabular}{|l|l|l|}
\hline Linear & 0.9733 & $94.74 \%$ \\
\hline Logarithmic-Y & 0.9724 & $94.56 \%$
\end{tabular}

square root- $X$

\begin{tabular}{|l|l|l|}
\hline Logarithmic-X & 0.9696 & $94.01 \%$ \\
\hline $\begin{array}{l}\text { Double } \\
\text { squared }\end{array}$ & 0.9518 & $90.59 \%$ \\
\hline
\end{tabular}

\begin{tabular}{|c|c|c|}
\hline squared & & \\
\hline Square root- $Y$ & 0.9499 & $90.23 \%$ \\
\hline
\end{tabular}

\begin{tabular}{|l|l|l|}
\hline $\begin{array}{l}\text { Squared-Y } \\
\text { logarithmic-X }\end{array}$ & 0.9255 & $85.65 \%$ \\
\hline
\end{tabular}

\begin{tabular}{|c|c|c|}
\hline logarithmic & & \\
\hline Exponential & 0.9165 & $83.99 \%$ \\
\hline
\end{tabular}

\begin{tabular}{l|l|l}
\hline Double & 0.9055 & $82.00 \%$
\end{tabular}

reciprocal

\begin{tabular}{l|l|l}
\hline Squared-X & 0.8940 & $79.93 \%$ \\
\hline
\end{tabular}

\begin{tabular}{|l|l|l}
\hline Square root- $Y$ & 0.8529 & $72.74 \%$
\end{tabular}

squared- $X$

\begin{tabular}{l|l|l}
\hline Reciprocal-Y & -0.8183 & $66.97 \%$ \\
\hline
\end{tabular}

\begin{tabular}{|l|l|l|}
\hline $\begin{array}{l}\text { Logarithmic-Y } \\
\text { squared-X }\end{array}$ & 0.8031 & $64.49 \%$ \\
\hline
\end{tabular}

\begin{tabular}{|l|l|l|}
\hline S-curve model & -0.8003 & $64.05 \%$ \\
\hline
\end{tabular}

\begin{tabular}{|l|l|l|}
\hline Reciprocal-X & -0.6920 & $47.89 \%$ \\
\hline
\end{tabular}

\begin{tabular}{|l|l|l}
\hline Reciprocal-Y & -0.6804 & $46.30 \%$
\end{tabular}

squared- $X$

\begin{tabular}{|l|l|l|}
\hline Squared-Y & -0.5994 & $35.92 \%$
\end{tabular}

reciprocal-X

Reciprocal-Y

square root- $\mathrm{X}$

Reciprocal-Y

logarithmic- $X$

\begin{tabular}{l|l}
\hline Square root-Y & $<$ no fit
\end{tabular}

reciprocal-X

\begin{tabular}{|l|l}
\hline Logistic & $<$ no fit $>$ \\
\hline
\end{tabular}

\begin{tabular}{|l|l}
\hline Log probit & $<$ no fi $>$
\end{tabular}

Sources: compiled by authors. 
S. Kvitka, G. Starushenko, V. Koval, H.Deforzh, O., Prokopenko. Marketing of Ukrainian higher educational institutions representation based on modeling of Webometrics Ranking

Table 3. The regression model of the world ranking of Ukrainian universities in January 2019

Simple Regression - World Rank January 2019 vs. Ukraine Rank January 2019

Comparison of Alternative Models

Dependent variable: World Rank January 2019

Independent variable: Ukraine Rank January 2019

Multiplicative model: $Y=a^{*} X^{\wedge} b$

\section{Coefficients}

\begin{tabular}{|l|l|l|l|l|}
\hline & Least Squares & Standard & $T$ & \\
\hline Parameter & Estimate & Error & Statistic & $P$-Value \\
\hline Intercept & 7.11314 & 0.0171756 & 414.141 & 0.0000 \\
\hline Slope & 0.408916 & 0.00554542 & 73.7394 & 0.0000 \\
\hline
\end{tabular}

NOTE: intercept $=\ln (\mathrm{a})$

Analysis of Variance

\begin{tabular}{|l|l|l|l|l|l|}
\hline Source & $\begin{array}{l}\text { Sum of } \\
\text { Squares }\end{array}$ & $D f$ & $\begin{array}{l}\text { Mean } \\
\text { Square }\end{array}$ & F-Ratio & $\begin{array}{l}P \text { - } \\
\text { Value }\end{array}$ \\
\hline Model & 6.47774 & 1 & 6.47774 & 5437.50 & 0.0000 \\
\hline Residual & 0.0571829 & 48 & 0.00119131 & & \\
\hline $\begin{array}{l}\text { Total } \\
\text { (Corr.) }\end{array}$ & 6.53492 & 49 & & & \\
\hline
\end{tabular}

Correlation Coefficient $=0.995615$

R-squared $=99.125$ percent

R-squared (adjusted for d.f.) $=99.1067$ percent

Standard Error of Est. $=0.0345154$

Mean absolute error $=0.0260793$

Durbin-Watson statistic $=0.627904(\mathrm{P}=0.0000)$

Lag 1 residual autocorrelation $=0.672326$

Plot of Fitted Model

World Rank January $2019=\exp \left(7,11314+0,408916^{*} \operatorname{In}(\right.$ Ukraine Rank January 2019)) (X 1000,0)

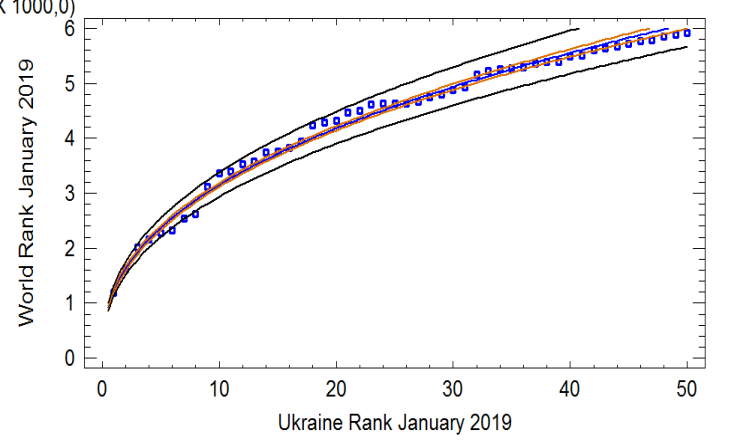

\begin{tabular}{|l|l|l|}
\hline Model & Correlation & $R$-Squared \\
\hline Multiplicative & 0.9956 & $99.12 \%$ \\
\hline $\begin{array}{l}\text { Squared-Y } \\
\text { square root-X }\end{array}$ & 0.9950 & $98.99 \%$ \\
\hline
\end{tabular}

\begin{tabular}{|l|l|l}
\hline Square root- $Y$ & 0.9933 & $98.67 \%$
\end{tabular}

\begin{tabular}{l|l} 
logarithmic-X & \\
\hline
\end{tabular}

\begin{tabular}{l|l|l}
\hline Square root- $X$ & 0.9928 & $98.56 \%$
\end{tabular}

\begin{tabular}{|l|l|l}
\hline Squared-Y & 0.9900 & $98.00 \%$ \\
\hline
\end{tabular}

\begin{tabular}{|l|l|l}
\hline Logarithmic- $X$ & 0.9823 & $96.48 \%$ \\
\hline
\end{tabular}

\begin{tabular}{|l|l|l|}
\hline $\begin{array}{l}\text { Double square } \\
\text { root }\end{array}$ & 0.9799 & $96.02 \%$ \\
\hline
\end{tabular}

\begin{tabular}{|l|l|l|}
\hline Linear & 0.9585 & $91.87 \%$ \\
\hline $\begin{array}{l}\text { Logarithmic-Y } \\
\text { square root- } X\end{array}$ & 0.9559 & $91.37 \%$ \\
\hline
\end{tabular}

\begin{tabular}{|c|c|c|}
\hline $\begin{array}{l}\text { Squared-Y } \\
\text { logarithmic-X }\end{array}$ & 0.9455 & $89.40 \%$ \\
\hline $\begin{array}{l}\text { Double } \\
\text { reciprocal }\end{array}$ & 0.9429 & $88.90 \%$ \\
\hline Double squared & 0.9299 & $86.47 \%$ \\
\hline Square root-Y & 0.9293 & $86.36 \%$ \\
\hline Exponential & 0.8887 & $78.98 \%$ \\
\hline Squared-X & 0.8660 & $75.00 \%$ \\
\hline S-curve model & -0.8415 & $70.82 \%$ \\
\hline $\begin{array}{l}\text { Square root- } Y \\
\text { squared- } X\end{array}$ & 0.8211 & $67.41 \%$ \\
\hline Reciprocal-Y & -0.7714 & $59.50 \%$ \\
\hline $\begin{array}{l}\text { Logarithmic-Y } \\
\text { squared-X }\end{array}$ & 0.7662 & $58.71 \%$ \\
\hline Reciprocal-X & -0.7294 & $53.20 \%$ \\
\hline $\begin{array}{l}\text { Squared-Y } \\
\text { reciprocal-X }\end{array}$ & -0.6339 & $40.18 \%$ \\
\hline $\begin{array}{l}\text { Reciprocal-Y } \\
\text { squared-X }\end{array}$ & -0.6301 & $39.70 \%$ \\
\hline $\begin{array}{l}\text { Reciprocal-Y } \\
\text { square root-X }\end{array}$ & $<$ no fit $>$ & \\
\hline $\begin{array}{l}\text { Reciprocal-Y } \\
\text { logarithmic-X }\end{array}$ & $<$ no fit $>$ & \\
\hline $\begin{array}{l}\text { Square root-Y } \\
\text { reciprocal-X }\end{array}$ & $<$ no fit $>$ & \\
\hline Logistic & $<$ no fit $>$ & \\
\hline Log probit & $<$ no fit $>$ & \\
\hline
\end{tabular}

Sources: compiled by authors. 
S. Kvitka, G. Starushenko, V. Koval, H. Deforzh, O. Prokopenko. Marketing of Ukrainian higher educational institutions representation based on modeling of Webometrics Ranking

Table 4. Conclusions from the simple regression calculations

\begin{tabular}{|c|c|c|}
\hline January 2018 & July 2018 & January 2019 \\
\hline \multicolumn{3}{|c|}{ Regression dependence of the world ranking of Ukrainian universities } \\
\hline$y_{1}=570.567+822.423 \sqrt{x},(2)$ & $y_{2}=516.728+792.407 \sqrt{x},(3)$ & $y_{3}=1227.997 x^{0.408916}(4)$ \\
\hline \multicolumn{3}{|c|}{ Determination coefficient } \\
\hline$R_{1}^{2}=99.3816$ & $R_{2}^{2}=99.1843$ & $R_{3}^{2}=99.125$ \\
\hline \multicolumn{3}{|c|}{$\begin{array}{l}\text { shows that the models are explained accordingly } 99.3816 \%, 99.1843 \% \text { and } 99.125 \% \text { changes in the } \\
\text { effective factor }\end{array}$} \\
\hline \multicolumn{3}{|c|}{ Correlation coefficient } \\
\hline$r_{1}=0.996903$ & $r_{2}=0.995913$ & $r_{3}=0.995615$ \\
\hline \multicolumn{3}{|c|}{ defines for all models the strong interconnection of variables } \\
\hline \multicolumn{3}{|c|}{ Standard error evaluation } \\
\hline 107.683 & 119.274 & 0.0345154 \\
\hline \multicolumn{3}{|c|}{ defines the standard deviation of the remnants for the models } \\
\hline \multicolumn{3}{|c|}{ Average absolute error } \\
\hline 87.9665 & 87.7746 & 0.0260793 \\
\hline \multicolumn{3}{|c|}{ is the average value of the remnants } \\
\hline \multicolumn{3}{|c|}{ Comparison of alternative models } \\
\hline \multicolumn{3}{|c|}{$\begin{array}{l}\text { The table shows the results of comparing the selected curvilinear regression models with the } \\
\text { alternatives. Models }(2)-(4) \text { are the best in comparison with the alternatives by the coefficient of } \\
\text { determination, because the value of statistics } R^{2} \text { in them is the most }\end{array}$} \\
\hline \multicolumn{3}{|c|}{ Schedule } \\
\hline $\begin{array}{r}\text { The graphs for each model depi } \\
\text { ba }\end{array}$ & $\begin{array}{l}\text { statistical data, regression curve, } \\
\text { ine data and regression curves. }\end{array}$ & nd confidence intervals for \\
\hline
\end{tabular}

Sources: compiled by authors.

Let's increase the expressions (1) - (2) by the variable $t$ (time parameter) with the help of the threepoint approximation of the Pade form:

$$
z_{[0 / 2]}(t)=\frac{a_{0}}{1+b_{1} t+b_{2} t^{2}}
$$

where $a_{0}, b_{i},(i=1,2)$ - constants on the variable $t$, which are determined from the conditions:

$$
\begin{aligned}
& \left.\frac{a_{0}}{1+b_{1} t+b_{2} t^{2}}\right|_{t=0}=y_{1} ; \\
& \left.\frac{a_{0}}{1+b_{1} t+b_{2} t^{2}}\right|_{t=6}=y_{2} ;
\end{aligned}
$$


that is:

$$
\left.\frac{a_{0}}{1+b_{1} t+b_{2} t^{2}}\right|_{t=12}=y_{3}
$$

$$
\begin{gathered}
a_{0}=570.567+822.423 \sqrt{x} \\
\frac{a_{0}}{1+6 \mathrm{~b}_{1}+36 b_{2}}=516.728+792.407 \sqrt{x} \\
\frac{a_{0}}{1+12 b_{1}+144 b_{2}}=1227.997 x^{0.408916}
\end{gathered}
$$

The solution of the system of equations (6), (7), obtained using the mathematical editor Maple, has the form:

$$
\begin{aligned}
& b_{1}=-\frac{44224.71052 x-76039.25 x^{0.9089159998}+59520.38678 \sqrt{x}-61007 x^{0.4089159998}+20007.37412}{x^{0.4089159998}\left(7.92407 \cdot 10^{5} \sqrt{x}+5.16728 \cdot 10^{5}\right)} \\
& b_{2}=\frac{7370.785088 x-11839.43056 x^{0.9089159998}+9920.064463 \sqrt{x}-8672.305556 x^{0.4089159998}+3334.562354}{x^{0.4089159998}\left(7.92407 \cdot 10^{5} \sqrt{x}+5.16728 \cdot 10^{5}\right)}
\end{aligned}
$$

Consequently, taking into account (6), (8), (9), the world rating $z$ Ukrainian Universities in the Internet Space by the System of Webometric Indicators, depending on the place of the university in the rating scale $x$ and time factor $t$ is described by the analytical relation of the species:

$$
z(x, t)=\frac{a_{0}}{1+b_{1} t+b_{2} t^{2}}
$$

Using the functions (5), (8), (9), (10) makes it possible: to obtain quantitative assessments and illustrate a qualitative picture of the rating of Ukrainian universities in the Internet space by Webometrix indicators during January 2018 - January 2019 (Fig. 1); to highlight the tendencies of the rating of domestic universities, showing its character in the forecast by July 2019 (Fig. 2). Charts in Figure 1 and Figure 2 are built in the mathematical editor Maple.

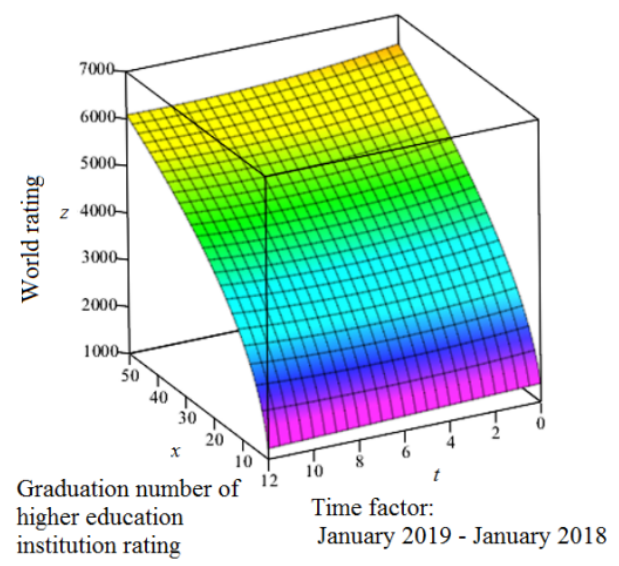

Figure 1. Webometric rating of Ukrainian universities in the period from January 2018 to January 2019 Sources: compiled by authors. 


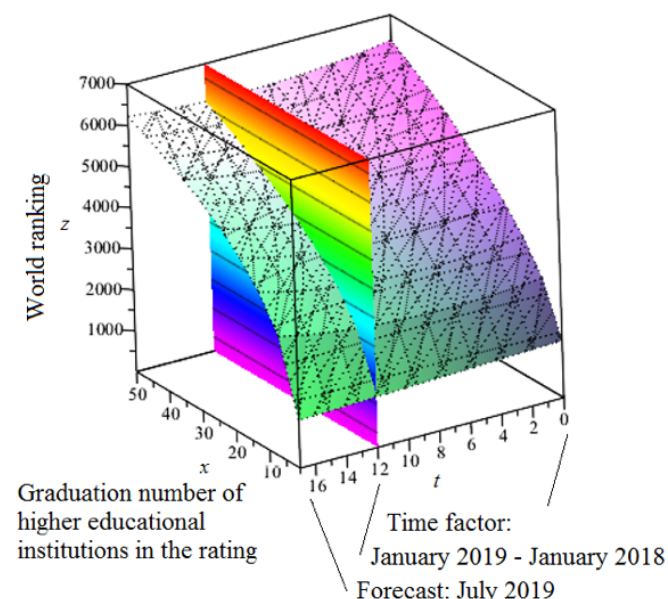

Figure 2. The rating of Ukrainian universities on the Webometric indexes from January 2018

Sources: compiled by authors.

by January 2019 and forecast by July 2019

The use of the relations (5), (8), (9), (10) obtained on the basis of the Pade approximation technique enables to calculate for the selected sample population the forecast of the average rating of Ukrainian universities in the Internet space in the Webometrix system of indicators.

For this we apply the expression (10), taking into account (5), (8), (9), the operator of averaging (Bakhvalov \& Panasenko, 1989):

$$
\tilde{z}(t)=\frac{1}{b-a} \int_{a}^{b} z(x, t) d x
$$

and use the STATGRAPHICS Forecast tool to analyze the data. The results of calculation are given in Table. 5

For forcasting, the model of one-parameter linear exponential smoothing of Brown with (Table 5 , Forecasting - Average Rank) is chosen. This model assumes that the best forecast for future data is determined by the linear trend, which is estimated by the exponential weight of all previous data values. According to error statistics (Table 5, Model Comparison) we find that the chosen model of one-parameter linear exponential smoothing of Brown (model E) is:

- Model with the lowest average square error (RMSE) during the assessment period;

- Model with the lowest average absolute error (MAE);

- Model with the lowest average absolute error (MAPE).

The selected model $E$ passes 3 tests out of 5 . Thus, according to the results of the analysis we find that the chosen one-parameter linear exponential smoothing model of Brown is the best among alternative models and can be used for prediction. The graph (Figure 3) is presented: source data for forecasting; graph of the function approximated by the model of one-parameter linear exponential smoothing of Brown; forecast of the average value of the world ranking of Ukrainian universities in the Internet space on the Webometrics system for 6 months (until July 2019 inclusive); Confidence intervals for the forecast at 95\% confidence level. 
S. Kvitka, G. Starushenko, V. Koval, H.Deforzh, O., Prokopenko. Marketing of Ukrainian higher educational institutions representation based on modeling of Webometrics Ranking

Table 5. Calculation forecast

\begin{tabular}{|c|c|c|}
\hline \multicolumn{3}{|c|}{ Forecasting - Average Rank } \\
\hline \multicolumn{3}{|c|}{ Data variable: Average Rank } \\
\hline \multicolumn{3}{|c|}{$\begin{array}{l}\text { Number of observations }=13 \\
\text { Start index }=1,0 \\
\text { Sampling interval }=1,0\end{array}$} \\
\hline \multicolumn{3}{|c|}{$\begin{array}{l}\text { Forecast Summary } \\
\text { Forecast model selected: } \\
\text { Brown's linear exp. smoothing } \\
\text { with alpha }=0,9999 \\
\text { Number of forecasts generated: } \\
6 \\
\text { Number of periods withheld for } \\
\text { validation: } 0\end{array}$} \\
\hline & Estimation & Validation \\
\hline RMSE & 22,922 & \\
\hline MAE & 14,5935 & \\
\hline MAPE & 0,329708 & \\
\hline ME & $\mid-1,7944$ & \\
\hline MPE & - $-0,035300$ & \\
\hline
\end{tabular}

The table below shows:

RMSE - mean square error;

MAE - average absolute error;

MAPE - average absolute

percentage error;

ME - mean error;

MPE is the average percentage error.

The first three statistics measure the magnitude of errors, and the best model gives it less value.

The last two statistics measure displacement; the best model gives it a value close to 0 .

Data variable: Average Rank

Number of observations $=13$; Start index $=1,0$;

Sampling interval $=1,0$

\section{Models}

(A) Random walk with drift $=-10,7901$

(B) Linear trend $=4443,18+-10,6179 t$

(C) Simple moving average of 3 terms

(D) Simple exponential smoothing with alpha $=0,9999$

(E) Brown's linear exp. smoothing with alpha $=0,9999$

\section{Estimation Period}

\begin{tabular}{|l|l|l|l|l|l|}
\hline Model & $R M S E$ & MAE & MAPE & ME & MPE \\
\hline$(\mathrm{A})$ & 26,8341 & 22,1791 & 0,506561 & $3,78956 \mathrm{E}-13$ & 0,00325043 \\
\hline$(\mathrm{B})$ & 49,8405 & 39,668 & 0,905677 & $-3,49806 \mathrm{E}-13$ & $-0,0108669$ \\
\hline$(\mathrm{C})$ & 45,2436 & 38,2963 & 0,879943 & $-16,2963$ & $-0,37576$ \\
\hline$(\mathrm{D})$ & 27,8677 & 21,5015 & 0,490388 & $-9,96087$ & $-0,225661$ \\
\hline$(\mathrm{E})$ & 22,922 & 14,5935 & 0,329708 & $-1,7944$ & $-0,035388$ \\
\hline
\end{tabular}

Key:

RMSE $=$ Root Mean Squared Error

RUNS = Test for excessive runs up and down

RUNM = Test for excessive runs above and below median

AUTO = Box-Pierce test for excessive autocorrelation

MEAN $=$ Test for difference in mean 1 st half to 2 nd half

$\mathrm{VAR}=$ Test for difference in variance 1 st half to $2 \mathrm{nd}$ half

OK $=$ not significant $(p>=0,05)$

${ }^{*}=$ marginally significant $(0,01<p<=0,05)$

$* *$ significant $(0,001<p<=0,01)$

${ }^{* * *}=$ highly significant $(p<=0,001)$

The table compares the results of five different forecasting models.

The table also contains the results of the five tests carried out on the residue to determine whether each model under consideration is adequate to the statistical data:

OK means that the model passes the test;

* means that the model does not pass the test at $95 \%$ confidence level;

${ }^{* *}$ means that the model does not pass the $99 \%$ trust test;

${ }^{* * *}$ mean that the model does not pass the test at the confidence level of $99.9 \%$. 
S. Kvitka, G. Starushenko, V. Koval, H. Deforzh, O. Prokopenko. Marketing of Ukrainian higher educational institutions representation based on modeling of Webometrics Ranking

Continued Table 5

\section{The table below shows:}

RMSE - mean square error;

MAE - average absolute error;

MAPE - average absolute

percentage error;

ME - mean error;

MPE is the average percentage error.

The first three statistics measure the magnitude of errors, and the best model gives it less value. The last two statistics measure displacement; the best model gives it a value close to 0 .

Sources: compiled by authors.

Time Sequence Plot for Average Rank

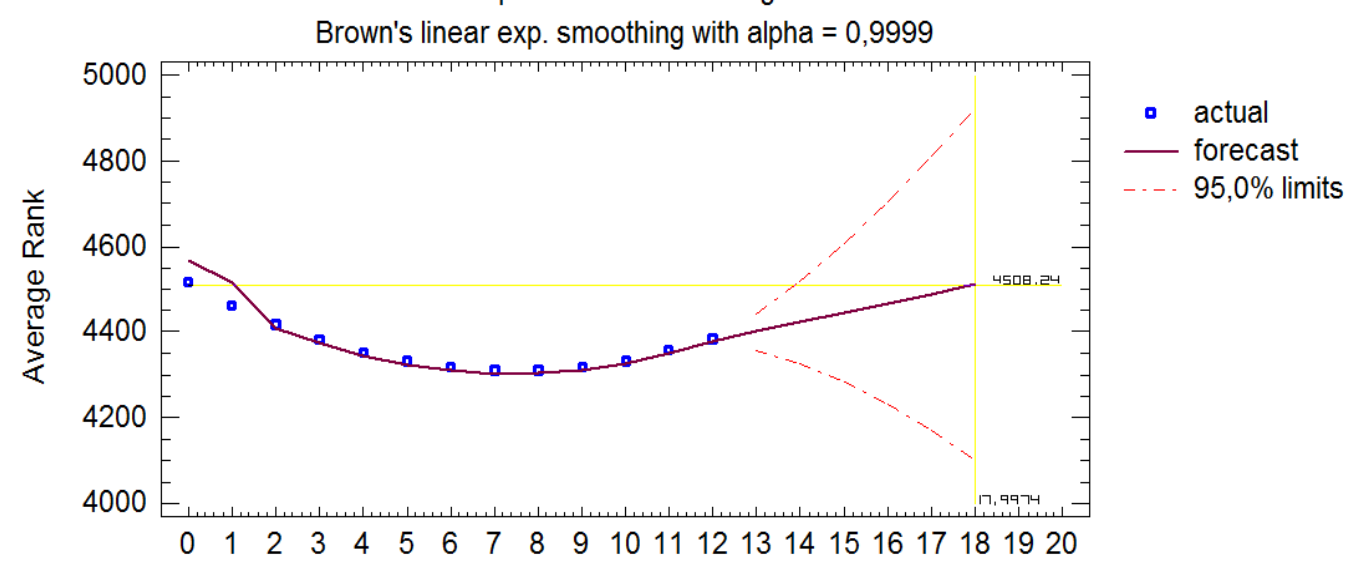

Figure 3. The forecast of the average world rating of the top 50 Ukrainian universities in the internet space for 6 months under the indicator system Webometrics

Sources: compiled by authors.

Thus, according to forecasted calculations at 95\% confidence level, the average value of the world ranking of the top 50 Ukrainian universities in the Internet space by indicators of Webometrics in July 2019 will slightly deteriorate compared with January 2019 and will be approximately $\hat{z}=4508 \pm 400$, that is, it will be in the range: $4108 \leq \hat{z} \leq 4908$.

Conclusions. On the basis of the statistical material of the Webometric rating of the top 50 Ukrainian universities during January 2018 - January 2019, a two-factor model that analytically describes the status of Ukrainian higher education institutions in terms of Webometrics, has been constructed, enables quantitative and qualitative analysis and forecasting of development trends. Statistical processing and analysis of the Webometric Ratings of Universities was conducted using the Professional Statistical Information Processing Program STATGRAPHICS Centurion XV.I. The analysis of regression and 
S. Kvitka, G. Starushenko, V. Koval, H.Deforzh, O., Prokopenko. Marketing of Ukrainian higher educational institutions representation based on modeling of Webometrics Ranking

predictive models and comparisons with alternative models is based on their adequacy to statistical data and acceptability for practical use.

Analytical relations on the model were obtained using the Pade approximation technique. Mathematical editor Maple has been used to perform mathematical transformations as well as visualize the results of research and illustrate a qualitative picture of the rating of Ukrainian universities in the Internet space using the Webometric indicators system. The proposed method of constructing a model can be extended to a larger sample of statistical material and a more significant time interval: its algorithm in this case will not undergo fundamental changes and complications. The presented methodology and data analysis algorithm allow for a generalization of the evaluation of rating systems of other nature: when assessing the educational and public work of students, in financial analysis, in conducting sociological tests and surveys, in medical biology studies and in other areas of public administration.

\section{References}

Baker, G. A., \& Graves-Morris, P. (1996). Pade approximants (2nd ed.). Cambridge, NY: Cambridge University Press.

Bakhvalov, N. S., \& Panasenko, G. P. (1989). Averaging processes in periodic media. Mathematical problems in mechanics of composite materials. Dordrecht: Kluwer.

Buinytska, O., Stepura, I., \& Smirnova, V. (2016). Webometrics Rating as Instrument for Quality Assessment of Open Educational E-environment University. An Open Educational E-environment of a Modern University, 2, 107-119.

Harvey, L. (2008). Rankings of higher education institutions: A critical review. Quality in Higher Education, 14(3), $187-207$.

Kochetkova, O., Vavilina, N., \&Charkina, O. (2016). Scientific and Educational Activities of Tertiary Institutions in Terms of International Indicators. Scientific and Technical Information, 2, 34-43.

Koval, V., Duginets, G., Plekhanova, O., Antonov, A., \& Petrova, M. (2019). On the supranational and national level of global value chain management. Entrepreneurship and Sustainability Issues, 6(4), 1922-1937.

Koval, V., Slobodianiuk, O., \& Yankovyi, V. (2018). Production forecasting and evaluation of investments using Allen two-factor production function. Baltic Journal of Economic Studies, 4(1), 219-226. doi.org/10.30525/2256-0742/2018-4-1-219-226.

Marginson, S. (2014). University rankings and social science. European Journal of Education, 49(1), 45-59.

Morze, N., \& Buinytska, O. (2015). Education Quality in Open E-environment Research. International Journal of Research in Elearning, 1(1), 27-46.

Nelipa, D., \& Batrymenko, O. (2018). Strategy, management model and educational scientific civil servants training program in postgraduate studies of the university. Naukovyi Visnyk NHU, 2, pp.178-183.

New Ranking Webometrics (2018a). Edition 2018.1.0. Retrieved from http://webometrics.info/en/Europe/Ukraine

New Ranking Webometrics (2018b). Edition 2018.2.1.3. Retrieved from http://webometrics.info/en/Europe/Ukraine

Novak, O., Levchenko, L., Levchenko, I., \& Kostenko, O. (2019). Features of Master's Degree Programs in Ukraine: Formation of Administrative Competencies. Naukovyi Visnyk NHU, 1, pp.141-146.

On the Higher Education (2014). Law of Ukraine no. 1556-VII dated July 1, 2014. Statements of Verkhovna Rada of Ukraine, 37-38, 2716

Porzionato M., \& De Marco F. (2015). Excellence and Diversification of Higher Education Institutions' Missions. In: Curaj A., Matei L., Pricopie R., Salmi J., Scott P. (eds). The European Higher Education Area. Springer, Cham.

Ranking Web of Universities (2019). Methodology. Retrieved from http://webometrics.info/en/Methodology

Rudenko, S., Sapenko, R., Bazaluk, O. \& Tytarenko, V. (2018). Management features of international educational projects between universities of Poland and Ukraine. Naukovyi Visnyk NHU, 2, 142-147.

Shin, J., Toutkoushian, R. (2011). The past, present, and future of university rankings. In University rankings. Theoretical basis, methodology and impacts on global higher education New York: Springer.

Starushenko, G., \& Bazylevskyi, S. (2018). Analysis of the International Ranking of Ukrainian Universities According to a Generalized Webometric Model in the Context of European Integration Processes in the Field of Education, 2018. Bulletin of Scientific Papers of Donetsk State University of Management, 19(305), 49-63.

Tiutiunnyk, A., \& Grytselyak, B. (2013). The Main Criteria and Indicators of the Webometrics Ranking of Universities in the World. Information Technology and Means of Teaching, 38(6), 234-244.

Westerheijden, D. F. (2012). Towards a Better Ranking in Higher Education and Research: Critique of global university rankings and an alternative. Higher education forum, 9, 77-87. 
S. Kvitka, G. Starushenko, V. Koval, H. Deforzh, O. Prokopenko. Marketing of Ukrainian higher educational institutions representation based on modeling of Webometrics Ranking

C.A. Квітка, D.Sc., доцент, Жешувська Політехніка ім. Ігнація Лукасевича, (Польща);

Г.А. Старушенко, D.Sc., доцент, Жешувська Політехніка ім. Іенація Лукасевича, (Польща);

В.В. Коваль, д.е.н, профресор, Одеський торговельно-економічний інститут Київського національного торговельно-економічного університету (Україна);

Г.В. Дефорж, д.і.н., доцент, Центральноукраїнський державний педагогічний університет імені Володимира Винниченка в м. Кропивницький (Україна);

О.В. Прокопенко, д.е.н, профессор, Талліннський технологічний університет (Естонія); Інноваційний університет Collegium Mazovia в м. Седльце (Польща).

Маркетинг представленості українських закладів вищої освіти на основі моделювання рейтингів у Вебометрікс

Метою статті є побудова на основі статистичного матеріалу Вебометричного рейтингу університетів двофракторної моделі, яка аналітично описує у часі стан українських вищих навчальних закладів за показниками Вебометрікс, що дає можливість його кількісного й якісного аналізу та прогнозування тенденцій розвитку ринку освітніх послуг та маркетингових досліджень у цій сфері. Статистичний аналіз даних Вебометричного рейтингу університетів проведено із застосуванням профессійної програми статистичної обробки інфоормації STATGRAPHICS Centurion XV.I. Аналітичні співвідношення отримані за допомогою техніки Паде-апроксимацій. Для візуалізаціі результатів дослідження та ілюстрації якісної картини рейтингу українських ВНЗ в Інтернетпросторі за системою Вебометричних індикаторів використано математичний редактор Марle. Побудовано двофакторну модель стану і прогнозу академічної представленості українських університетів за даними Вебометричного рейтингу. Із застосуванням пропонованої моделі отримано аналітичний вираз, що дає змогу кількісної й якісної оцінок світового рейтингу вищих навчальних закладів України в системі Веб-індикаторів Вебометрікс щодо збільшення їх академічної присутності в Інтернеті, зміцнення міжнародного авторитету і підняття в цілому вітчизняної наукової школи на якісну нову ступінь розвитку. Наукова новизна роботи полягає у включенні в модель часового чинника, наявність якого дає можливість, по-перше, розширювати у часі використовуваний статистичний матеріал і, по-друге, прогнозувати тенденції і перспективи представлення в Інтернет-просторі університетів України як передових науково-дослідницьких центрів. Основні результати роботи можуть бути корисними в якості методичного матеріалу при проведенні учбового процесу у вищих навчальних закладах, при навчанні і підвищенні кваліфікації управлінських кадрів, при розробці програм реформування освітнього процесу у вищій школі з метою наближення його до європейського вимог і досягнення рівня найкращих світових університетів. Представлена методика й алгоритм аналізу даних можуть бути узагальнені для оцінки рейтингових систем іншої природи: при оцінюванні навчальної та громадської роботи учнів $і$ студентів, у фрінансовій аналітиці, зокрема, для оцінки ймовірності дефолту некредитних організацій, при визначенні надійності комериійних банків, при проведенні соціологічних тестів і опитувань, в медико-біологічних дослідженнях та в інших сфрерах публічного управління.

Ключові слова: заклад вищої освіти, університет, вебометричний рейтинг, апроксимація Паде, двухфакторная модель.

Manuscript received: 25.07.2019.

(c) The author(s) 2019. This article is published with open access at Sumy State University. 